\title{
An unusual case of hyponatraemia in diabetic ketoacidosis
}

\author{
P J Twomey, J Cordle, D R Pledger, Y Miao
}

J Clin Pathol 2005;58:1219-1220. doi: 10.1136/icp.2005.025916

This report outlines a case of diabetic ketoacidosis associated hyponatraemia in an 18 year old woman with type 1 diabetes who presented to the accident and emergency department and was quickly admitted to the intensive treatment unit. Causes of hyponatraemia include sodium depletion, pseudohyponatraemia, and extracellular hypertonicity. Hypertonicity secondary to hyperglycaemia is thought to be the major cause of hyponatraemia in diabetic ketoacidosis. Indirect and direct sodium measurements were performed until the glucose concentration stabilised. The large difference between the presenting sodium concentrations is consistent with pseudohyponatraemia. However, the causes of pseudohyponatraemia (large increases in total protein, triglyceride, and cholesterol concentrations) were excluded. Analytical error should always be considered when the laboratory results do not agree with the clinical picture. Sometimes, however, even after excluding all known effects, the cause may remain unexplained, as in this case.

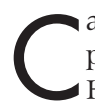
auses of hyponatraemia include sodium depletion, pseudohyponatraemia, and extracellular hypertonicity. Hypertonicity ${ }^{1}$ secondary to hyperglycaemia is believed to be the major cause of hyponatraemia in diabetic ketoacidosis (DKA). ${ }^{2}$ We outline a case of DKA associated hyponatraemia in an 18 year old patient with type 1 diabetes who presented to the accident and emergency department and was quickly admitted to the intensive treatment unit. Indirect (Olympus AU600) and direct (AVL 9180 Electrolyte Analyser, calibrated to give the same result as one would expect from indirect methods) sodium measurements were performed until the glucose concentration stabilised. The initial indirect sodium was confirmed by two further analyses and the direct sodium confirmed by a further single analysis; the internal quality control values before and immediately after these analyses were within acceptable limits and all analytes performed satisfactorily at this time on proficiency testing. Thus, the large difference between the presenting sodium concentrations is consistent with pseudohyponatraemia (table 1).

Pseudohyponatraemia occurs with decreases in the electrolyte containing aqueous phase of serum/plasma. Ion specific electrodes (ISEs) only measure activity in this phase. Accordingly, when a direct ISE gives an activity of $140 \mathrm{mmol} /$ litre in a specimen where triglycerides make up $20 \%$ of the total volume, an indirect method that has a dilution of one part specimen to nine parts water would dilute the nonaqueous phase to $2 \%$ of the total volume. Thus, the aqueous phase makes up $98 \%$ of the diluted sample, giving an activity of cl $1.43 \mathrm{mmol} /$ litre $((140 \times 0.8) /(10 \times 0.98))$, which is multiplied up to $114.3 \mathrm{mmol} / \mathrm{litre}$, thus creating a $26 \mathrm{mmol} /$ litre difference between the two methods. Causes of pseudohyponatraemia include large increases in total protein and triglyceride concentrations and rarely hypercholesterolaemia when lipoprotein $\mathrm{X}$ is formed as a result of primary biliary cirrhosis. ${ }^{3}$ Because hypertriglyceridaemia and hyperproteinaemia are not rare in clinical practice, sodium methods that do not contain a dilution step are preferable. ${ }^{4}$ However, many routine analysers still use indirect methods, because they require smaller sample volumes.

Potential pseudohyponatraemia attributable to serum triglyceride and total protein was calculated using Waugh's empirical equation assuming that normal serum/plasma water is $93 \% .^{5}$ A positive value implies that sodium measured by the indirect ISE should be lower than the direct ISE by the given amount, and vice verse for negative values. The contribution of extracellular glucose to hyponatraemia has also been empirically modelled ${ }^{6}$ and more recently measured using an indirect methodology. ${ }^{7}$ Using these equations, ${ }^{5-7}$ we calculated the predicted stable serum sodium concentration (table 1).

\section{"It is important to elucidate the cause(s) of hyponatraemia to ensure the appropriate interpretation of results and that the appropriate therapeutic action is taken"}

Our case has two unusual features. First, the pseudohyponatraemia is not explained by the triglyceride and total protein concentrations and disappeared when the glucose returned to the euglycaemic range. Second, the older Katz equation $^{6}$ proved to be the most accurate when the indirect method was used to estimate the euglycaemic sodium concentration. This raises several questions: (1) does DKA have an addition effect on either sodium metabolism or sodium measurement in addition to the effect of hyperglycaemia alone as recently assessed by Hillier? There are theoretical analytical reasons to believe that low bicarbonate concentrations and acidosis may result in a relatively lower indirect sodium than expected when compared with a direct measurement. ${ }^{8}$ (2) It would be expected that the Katz equation would work best with the direct sodium because this equation was empirically modelled. (3) Are these equations valid using modern technology-the Hillier paper used the non-routine flame photometry methodology. (4) The calibration of direct ISEs may only be valid in "normal" sera. (5) Such population based equations may not perform satisfactorily in each individual situation.

Ideally, physicians should know the sodium method used by their laboratory. However, it is unlikely that they will always know or appreciate associated issues, so that it may sometimes be necessary for the chemical pathologist to facilitate optimal patient care, ${ }^{9}$ especially when significant hypertriglyceridaemia or hyperproteinaemia exists. It is important to elucidate the cause(s) of hyponatraemia to ensure the appropriate interpretation of results and that the appropriate therapeutic action is taken. Analytical error should always be considered when the laboratory results do not agree with the clinical picture. Sometimes, however, even

Abbreviations: DKA, diabetic ketoacidosis; ISE, ion specific electrode 
Table 1 Analyte concentrations at presentation and after stabilisation of the glucose concentration

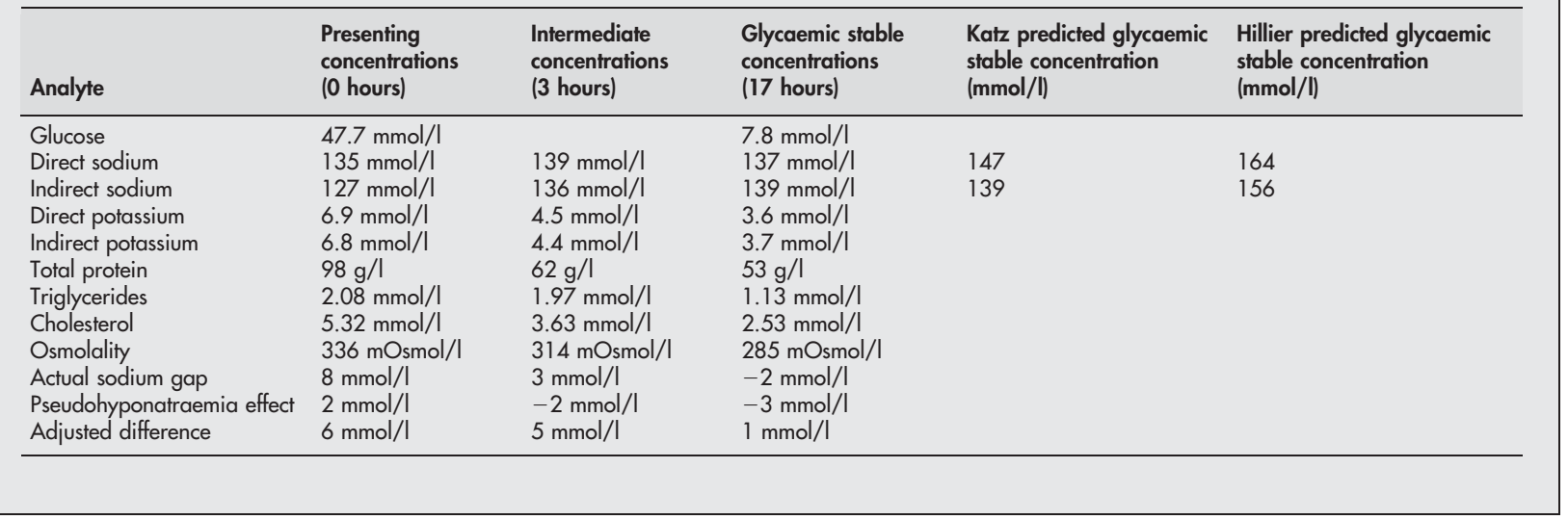

after excluding all known effects, the cause may remain unexplained. In addition, this case raises the question of the validity of derived equations using modern technology and we suggest that further research is carried out to answer this question in patients with DKA and hyperosmolar non-ketotic hyperglycaemia.

\section{Authors' affiliations}

P J Twomey, J Cordle, D R Pledger, Department of Clinical Biochemistry, The lpswich Hospital, Heath Road, Ipswich IP4 5PD, UK

Y Miao, Department of Gastroenterology and General Medicine, The Ipswich Hospital

Correspondence to: Dr P J Twomey, Department of Clinical Biochemistry, The Ipswich Hospital, Heath Road, Ipswich IP4 5PD, UK; PatTwomey@doctors.org.uk

Accepted for publication 2 March 2005

\section{REFERENCES}

1 Seldin DW, Tarail R. Effect of hypertonic solutions on metabolism and excretion of electrolytes. Am J Physiol 1949;159:160-74.

2 Walmsley RN, Watkinson LR, Koay ESC. Cases in chemical pathology-a diagnostic approach, 3rd ed. Singapore: World Scientific.

3 Ko GTC, Yeung VTF, Chow C-C, et al. Pseudohyponatraemia secondary to hypercholesterolaemia. Ann Clin Biochem 1997;34:324-5.

4 National Committee for Clinical Laboratory Standards. Standardization of sodium and potassium ion-selective electrode systems to the flame photometric reference method: approved standard C29-A. Wayne, PA: National Committee for Clinical Laboratory Standards, 1995.

5 Waugh WH. Utility of expressing serum sodium per unit of water in assessing hyponatremia. Metabolism 1969;18:706-12.

6 Katz M. Hyperglycemic-induced hyponatremia-calculation of expected serum sodium depression. N Engl J Med 1973;289:843-4.

7 Hillier TA, Abbott RD, Barrett EJ. Hyponatremia: evaluating the correction factor for hyperglycaemia. Am J Med 1999; 106:399-403.

8 Apple FS, Koch DD, Graves S, et al. Relationship between directpotentiometric and flame-photometric measurement of sodium in blood. Clin Chem 1982;28:1931-5.

9 Crook $M$. The investigation and management of severe hyponatraemia. J Clin Pathol 2002;55:883. 